Research Article

\title{
Optimization of Silylation for Parabens Determination by Gas Chromatography-Mass Spectrometry
}

\author{
Rosalina Djatmika ${ }^{1,2^{*}}$, Wang-Hsien Ding ${ }^{2}$ \\ 1 Department of Chemistry, Faculty of Mathematics and Natural Sciences, Brawijaya University, Malang, Indonesia \\ ${ }^{2}$ Department of Chemistry, National Central University, 300 Jhong-Da Road, Jhong-Li, Taoyuan, Taiwan
}

\section{ARTICLE INFO}

Article history

Revised 29 September 2016

Accepted 24 October 2016

Available online 1 November 2016

*Author corresponding

Email: rosalina.djatmikaa@gmail.com

\begin{abstract}
A low cost, environmental friendly and convenient method for parabens derivatization using Gas Chromatography-Mass Spectrometry (GC-MS) analysis is investigated. Derivatization is needed to enhance the thermal stability, detectability, and volatility of parabens to make them amenable for gas chromatographic analysis. This method involved on-line derivatization by silylating reagent: N-Methyl-N-tertbutyldimethylsilyltrifluoroacetamide (MTBSTFA), N,O-bis (trimethylsilyl) trifluoroacetamide (BSTFA), N-methyl-N-trimethylsilyltrifluoroacetamide (MSTFA), and $\mathrm{N}, \mathrm{O}-\mathrm{bis}$ (trimethylsilyl)acetamide (BSA). The variables affected the derivatization process, such as, types and volumes of silylating reagents, injection port temperature, and purge-off time, were evaluated to obtain the optimal condition for determination of parabens. The Relative Response Factors (RRF) was used as a parameter of parabens derivatization efficiency to obtain the best compromise condition of each variable. On a comprehensive level, a comparison of the optimal condition of each silylating reagent was evaluated. Moreover, $1 \mu \mathrm{L}$ of MSTFA at $260^{\circ} \mathrm{C}$ of injection port temperature and $2.5 \mathrm{~min}$ purge-off time (in splitless mode) obtained the most effective of derivatization process.
\end{abstract}

Keywords: Parabens, on-line silylation, Gas Chromatography-Mass Spectrometry

\section{Introduction}

Parabens or esters of p-hydroxybenzoic acid that include methylparaben (MP), ethylparaben $(\mathrm{EP})$, propylparaben (PP) and butylparaben (BP) are commonly used as antimicrobial preservatives in cosmetics, food, industrial and pharmaceuticals products due to their low toxicity, low cost, inert nature and worldwide regulatory acceptance (Han, Xia, Chen, Shen, Miao, \& Shen, 2016). The widespread use of parabens causes their ubiquitous existence in environment. Parabens have been found in indoor dust (Tran, Minh, Kumosani, \& Kannan, 2016), surface water (Rocio-
Bautista et al., 2015), soil, sediment/sludge (Ferreira, Moder, \& Laespada, 2011), cosmetics and personal care products (Rodas, Portugal, Avivar, Estela, \& Cerda, 2015), seafood (Han et al., 2016), and other foodstuff (Liao, Chen, \& Kannan, 2013). The finding parabens in human urine (Moos et al., 2015), blood, breast milk (Azzouza, Rascon, \& Ballesteros, 2016) and serum (Hines, Mendola, van Ehrenstein, Ye, Calafat, \& Fenton, 2015) proved that human have been exposed parabens. Although parabens have been considered low acute toxicity compounds, but controversy about side effect of parabens arose due to their effects on the endocrine system. Previous studies have 
reported that parabens have endocrine disruptive effect which cause human male reproductive disorders and play a role in enhance the risk of breast cancer (Shanmugam, Ramaswamy, Radhakrishnan, \& Tao, 2010). Because of parabens have potential health effects of endocrine disrupting factors, developing method for parabens analysis got great interest.

Gas chromatography-mass spectrometry (GCMS) is commonly used for identification and separation parabens. To improve GC chromatographic separation, derivatization is typically used to increase the volatility of parabens and to improve sensitivity (Bowden, Colosi, MoraMontero, Garret, \& Yost 2009). However, derivatization reactions commonly are performed off-line, but it needed multi-step reactions, long time-consuming, and used toxic reagents (Wang, $\mathrm{Ma}, \quad Y i n, \quad \& \quad \mathrm{Xu}, \quad 2013)$ On-line derivatization reduces solvent waste, simplifies sample preparation, and avoids the need for hazardous reagents ( $\mathrm{Wu}, \mathrm{Hu}$, Yue, Yang, \& Zhang, 2009).

On-line silylation was a derivatization using silylation reagent which developed and applied for the analysis of parabens with GC-MS. Silylation is the most commonly used in derivatization procedures for GC-MS analysis since it could improve the GC sensitivity, accuracy and resolution by enhancing thermostability, detectability and decreasing peak tailing (Wang et al., 2013). The active hydrogen on hydroxyl groups of parabens is replaced with silyl groups of the silylation reagent during derivatization process. There are many kinds of silylation reagents, such as N-Methyl-Ntert-butyldimethylsilyltrifluoroacetamide (MTBSTFA), $\mathrm{N}, \mathrm{O}$-bis (trimethylsilyl) trifluoroacetamide (BSTFA), N-methyl-N-

trimethylsilyltrifluoroacetamide (MSTFA), and N,Obis(trimethylsilyl)acetamide (BSA) which play important role in the derivatization efficiency. The derivatization factors affected derivatization efficiency, such as the types and volumes of silylating reagent, injection port temperature, and purge-off time were also investigated.

\section{Materials and methods}

\subsection{Materials}

All chemicals are analytical grade and used without further purification: methylparaben (Alfa Aesar), ethylparaben (Alfa Aesar), propylparaben
(Alfa Aesar), butylparaben (Alfa Aesar), pTerphenyl- $\mathrm{d}_{14}$ as an internal standard (SigmaEldrich), dichloromethane (Macron), methyl alcohol (Merck), acetone (Macron), acetic anhydride (Sigma-Aldrich), and deionized water, Milli-Q water produced by Millipore Elix $10 \mathrm{RO}$ system and a Millipore Synergy UV system. The silylation derivatization reagents used: N-Methyl-Ntertbutyldimethylsilyltrifluoroacetamide (MTBSTFA), N,O-bis(trimethylsilyl)trifluoroacetamide (BSTFA), N-methyl-N-trimethylsilyl-

trifluoroacetamide (MSTFA), and N,O-bis(trimethylsilyl)-acetamide were purchased from Sigma-Aldrich.

\subsection{Preparation of stock solutions}

All solution used in this experiments was prepared using microsyringes to measure accurately which conditioned before by rinsing the syringes with dichloromethane, acetone, and methanol. The methylparaben stock solution was made by dissolving $10 \mathrm{mg}$ of methylparaben solid standard with $10 \mathrm{~mL}$ methanol to make $1000 \mathrm{ppm}$ of methylparaben (MP). The same procedure was applied to prepare ethylparaben (EP), propylparaben (PP), and butylparaben (PP) stock solutions. The internal standard stock solution was prepared by dissolving $10 \mathrm{mg}$ of internal standard with $10 \mathrm{~mL}$ dichloromethanes. These stock solutions were stored at $4^{\circ} \mathrm{C}$ in the dark to prevent degradation by light. A stock solution of internal standard was prepared by dissolving $\mathrm{mg}$ of $10 \mathrm{mg}$ $p$-Terphenyl- $d_{14}$ in $10 \mathrm{~mL}$ dichloromethane to make 1000 ppm.

\subsection{Preparation of working solutions}

The working solution was prepared by diluting stock solution. To prepare MP, EP, PP, BP, and internal standard working solution, the stock solutions of parabens standard was diluted with methanol and internal standard stock solution was diluted with dichloromethane until reach concentration 1.0 ppm of MP, EP, PP, and BP.

\subsection{GC-MS analysis}

Analysis was performed by gas chromatography-mass spectrometry (GC-MS) system: Varian $450 \mathrm{GC}$ directly connected to a Varian 220 ion-trap mass spectrometer (Walnut Creek, CA, USA) operated in electron ionization (EI) which set to full scan at mass range 100-500 $\mathrm{m} / \mathrm{z}$ (at $70 \mathrm{eV}$ electron energy). Gas 
chromatography column used was DB-5MS capillary column $(30 \times 0.25 \mathrm{~mm}$ i.d., $0.25 \mu \mathrm{m}$ film thickness). A ChromatoProbe (Varian) was used to introduce large-volume samples for on-line silylation. High purity helium $(99.999 \%)$ at a flow rate of $1 \mathrm{~mL} / \mathrm{min}$ was used as carrier gas.

The transfer line and ion source temperatures were set to 250 and $180^{\circ} \mathrm{C}$, respectively. Oven temperature was programmed to begin at $100^{\circ} \mathrm{C}$ for $4.0 \mathrm{~min}$, increased by $25^{\circ} \mathrm{C} / \mathrm{min}$ to $300^{\circ} \mathrm{C}$ and maintained for $3 \mathrm{~min}$. The injection-port temperature was held at $260^{\circ} \mathrm{C}$ for $2.5 \mathrm{~min}$ in order to complete silylation reaction and solvent vaporisation, then the temperature was accelerated rapidly to $300^{\circ} \mathrm{C}$ for silylated derivatives introduction into the analytical column.

\subsection{On-line silylation procedure}

Each sample solution contained standard mixed of 4 types of parabens (methylparaben, ethylparaben, propylparaben and butylparaben). In a typical run, $10 \mu \mathrm{L}$ volume of standard parabens mixed containing internal standard solution was added various silylating reagent in different volume. The mixing solution then introduced into a microvial, the vial was took into a ChromatoProbe vial holder, and then placed in the GC injection-port to obtain on-line silylation. Important silylation parameters, such as types and volumes of silylating reagent, injection port temperature and purge-off time were investigated to obtain the optimal condition of silylation process.

\section{Result and discussion}

\subsection{GC-MS analysis of on-line silylation}

Parabens are polar and thermally fragile compounds thus need derivatization to convert into more volatile compound for GC-MS analysis. Derivatization turned them into less polar, thermally stable and more volatile compounds for GC-MS analysis. Silylation is a derivatization procedure which substitute active hydrogen of the hydroxylated molecule with a silyl group of silylating reagent. Silylation reagents will convert hydroxyl alcohol to form tertiobutyldimethylsylil- (TBDMS) ethers (for derivatizations with MTBSTFA) and trimethylsilyl (TMS) ether (for derivatizations with $\mathrm{BSTFA}$ ) via $\mathrm{SN}_{2}$ substitution reaction, resulting a derivative for each compound. The silylated derivatives formed are volatile and for the most part, are easily separated (Scott, 2003). Fig. 1 displays the reactions of parabens with various silylating reagents (MTBSTFA, MSTFA, BSTFA, and BSA). The success of on-line silylation was evaluated with the appeared of molecular ion of silylated derivatives. Table 1 summarises the molecular ion and the fragment ions for each silylated derivatives observed in mass spectra.

a)<smiles>[R]OC(=O)c1ccc(O[Si](C)(C)C(C)(C)C)cc1</smiles>

b)

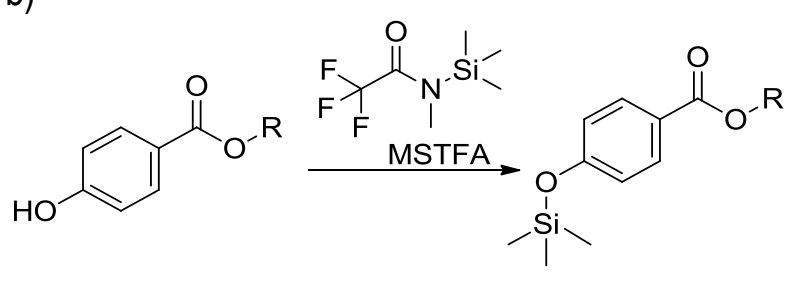

c)<smiles>[R]OC(=O)c1ccc(O[Si](C)(C)C(=N[Si](C)(C)C(=N[Si](C)(C)C)C(F)(F)O[Si](C)(C)C)c2ccc(O)cc2)cc1</smiles>

d)<smiles>[R]OC(=O)c1ccc(O[Si](C)(C)C(C)(C)C(=O)O[Si](C)(C)C)cc1</smiles>

Fig. 1. The reactions of parabens with various silylating reagents: a) MTBSTFA, b) MSTFA, c) BSTFA, d) BSA.

Molecular ions $\left([\mathrm{M}]^{+}\right)$showed in Table 1 is the molecular ion of silylated derivatives of each analyte. For MTBSTFA derivatization, [M-57] is corresponding to the loss of tert-butyl group of 
MTBSTFA-derivatives. Whereas for MSTFA, BSTFA, and BSA derivatization, [M-15] is corresponding to the loss of methyl group. The loss of tert-butyl and methyl group resulting silylated derivatives possessed good thermal and hydrolytic stability (Wang et al., 2013). Ion at $195 \mathrm{~m} / \mathrm{z}$ $\left(\left[\left(\mathrm{CH}_{3}\right)_{3} \mathrm{SiO}-\mathrm{Ar}-\mathrm{CO}+2 \mathrm{H}\right]^{+}\right)$observed in all of molecule was confirmed by loss of tert-butyl group (for MTBSTFA derivate) or $-\mathrm{CH}_{3}$ group (for other silylated derivatives) of the $\mathrm{Si}\left(\mathrm{CH}_{3}\right)_{3}$ and by loss of $-\left(\mathrm{CH}_{3}\right) n$ ( $n=1$ to 4 for MP, EP, PP, and BP). The intense signals detected in MS spectra were ions of $\mathrm{m} / \mathrm{z} 151,163$ and 177 which attributed to the loss of $\mathrm{CO}_{2}$ (carbondioxide), $\mathrm{O}_{2}$ (oxygen) and $\mathrm{H}_{2} \mathrm{O}$ (water), respectively.
Table 2.

The optimal condition of each silylating reagent

\begin{tabular}{lccc}
\hline $\begin{array}{l}\text { Silylating } \\
\text { Reagent }\end{array}$ & $\begin{array}{c}\text { Temperature } \\
\text { of Injection- } \\
\text { port }\left({ }^{\circ} \mathrm{C}\right)\end{array}$ & $\begin{array}{c}\text { Derivatization } \\
\text { Time }(\mathrm{min})\end{array}$ & $\begin{array}{c}\text { Silylating } \\
\text { Reagent } \\
\text { Volume } \\
(\boldsymbol{\mu L})\end{array}$ \\
\hline MSTFA & 260 & 2.5 & 1.0 \\
BSA & 300 & 3.0 & 2.0 \\
BSTFA & 280 & 2.5 & 2.0 \\
MTBSTFA & 300 & 2.0 & 1.0 \\
\hline
\end{tabular}

Table 1.

The molecular and the fragment ions for each silylated derivatives observed in mass spectra.

\begin{tabular}{ccccc}
\hline Silyl Reagents & Analytes & {$[\mathrm{M}]^{+}$} & {$[\mathrm{M}-\mathbf{5 7}] /[\mathrm{M}-\mathbf{1 5}]$} & Fragment ions \\
\hline MTBSTFA & Methylparaben & 266 & 209 & $195,177,151$ \\
& Ethylparaben & 280 & 223 & $195,177,163,151$ \\
& Ethylparaben & 294 & 237 & $195,163,151$ \\
& Butylparaben & 308 & 251 & $210,195,151$ \\
& & & \\
MSTFA & Methylparaben & 224 & 209 & $195,177,151$ \\
& Ethylparaben & 238 & 223 & $195,177,163,151$ \\
& Ethylparaben & 252 & 237 & $195,163,151$ \\
& Butylparaben & 266 & 251 & $210,195,151$ \\
BSTFA & & & \\
& Methylparaben & 224 & 209 & $195,177,151$ \\
& Ethylparaben & 238 & 223 & $195,177,163,151$ \\
& Ethylparaben & 252 & 237 & $195,163,151$ \\
& Butylparaben & 266 & 251 & $210,195,151$ \\
& BSA & & & \\
& Methylparaben & 224 & 209 & $195,177,151$ \\
& Ethylparaben & 238 & 223 & $195,177,163,151$ \\
& Ethylparaben & 252 & 237 & $195,163,151$ \\
& Butylparaben & 266 & 251 & $210,195,151$ \\
\hline
\end{tabular}

Table 3.

The RRF of the optimal condition for each silylation reagent

\begin{tabular}{lcccc}
\hline \multicolumn{1}{c}{ Silylating Reagent } & MP & EP & PP & BP \\
\hline MSTFA & $45.06 \pm 0.03$ & $40.45 \pm 0.07$ & $81.02 \pm 0.05$ & $93.64 \pm 0.06$ \\
BSA & $66.38 \pm 0.02$ & $37.60 \pm 0.09$ & $27.91 \pm 0.06$ & $21.86 \pm 0.05$ \\
BSTFA & $53.74 \pm 0.05$ & $50.53 \pm 0.03$ & $80.07 \pm 0.14$ & $84.44 \pm 0.09$ \\
MTBSTFA & $6.32 \pm 0.11$ & $11.73 \pm 0.01$ & $29.41 \pm 0.03$ & $19.06 \pm 0.03$ \\
\hline
\end{tabular}




\subsection{Optimisation of the silylation}

Optimisation of the silylation was evaluated using standard mixed of parabens consisted of examining injection-port temperature, purge-off time, and volume of silylating reagent with various silylating reagents: MTBSTFA, MSTFA, BSTFA, and BSA. The optimal condition of injection-port temperature, purge-off time, and volumes of silylating reagent of each reagent was used for comparison, as shown in Table 2. The RRF was used as a derivatization efficiency parameter to evaluate the best compromise condition of each variable. Table 3 shows RRF of the optimal condition for each silylating reagent.

According to Table 2 and Table 3, MSTFA and BSTFA as silylating reagents have good RRF due to both are appropriate reagent choice for derivatization of hydroxylated compounds (Bowden et al., 2009). Otherwise, MSTFA offers better compromise condition than BSTFA since it had higher RRF values than others and only required $1.0 \mu \mathrm{L}$ of MSTFA reagent for 2.5 min for silylation reaction and offers acceptable reproducibility. This result was corresponding with previous study mentioned that MSTFA is more potent and selective trimethylsilyl donor for reactions, mainly with $\mathrm{OH}$ groups, thus it could give good RRF for parabens derivatization (Wu, \& Lee, 2006).

\subsection{Injection-port temperature}

Injection-port temperature had effect to the derivatization efficiency as it thermally catalyse the derivatization reaction process. The various injection-port temperatures ranging from 220 to $320^{\circ} \mathrm{C}$ (at $20^{\circ} \mathrm{C}$ increments) were examined at a 2.5 min purge-off time. As shown in Figure 3, the RRF values of TMS-derivatives enhanced gradually when the injection-port temperature was increased from $220-260^{\circ} \mathrm{C}$, but thereafter it decreased significantly. Therefore, in this study, $260^{\circ} \mathrm{C}$ was chosen as the optimised injection-port temperature. It was corresponding with some previous study which reported that the injection-port was set between 250 to $300^{\circ} \mathrm{C}$ to obtain fast and completely reaction (Wang et al., 2013). The high temperature could overcome the energy barrier of the reaction and steric interference, thus made increasing of the reaction efficiency ( $\mathrm{Wu} \&$ Lee, 2006). Otherwise, a too high temperature could reduce analytical signals due to derivatives decomposition (Wang et al., 2013). In the other side, a low injection-port temperature resulted the low of GC signal because the analytes did not derivatized completely.

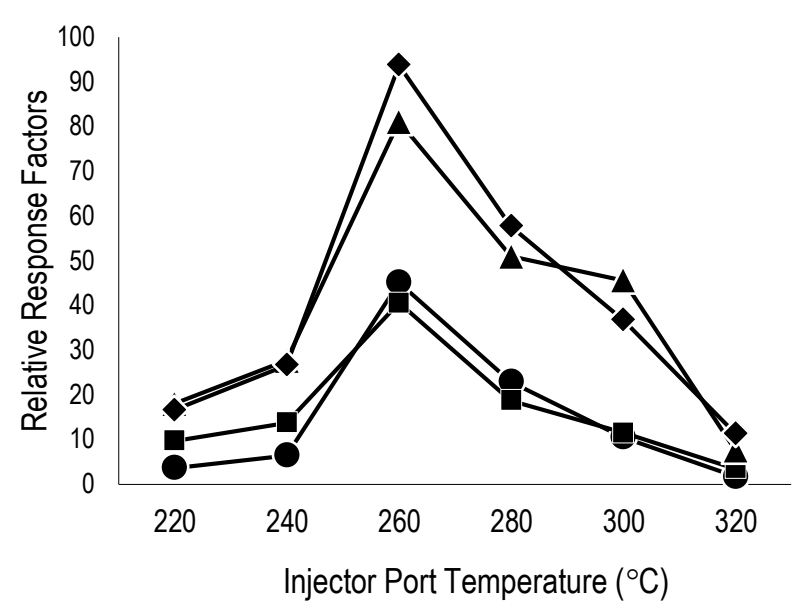

Fig. 3. Effect of injection-port temperature. $\operatorname{MP}(\bullet)$, EP $(\boldsymbol{\bullet}), \operatorname{PP}(\boldsymbol{\Delta})$ and $\operatorname{BP}(\bullet)$.

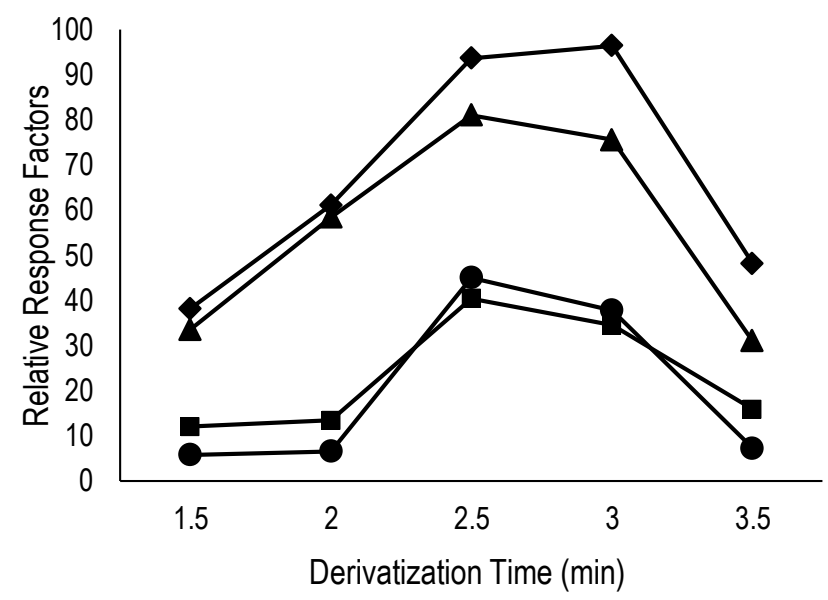

Fig. 4. Effect of the time of silylating. $\operatorname{MP}(\bullet), \operatorname{EP}(\boldsymbol{\bullet})$, $\operatorname{PP}(\boldsymbol{\Delta})$, and $\operatorname{BP}(\bullet)$.

\subsection{Purge-off time}

Other parameter influenced the on-line silylation process is the purge-off time, which evaluated for $1.5,2.0,2.5,3.0$, and $3.5 \mathrm{~min}$ in splitless mode with injection-port temperature at $260^{\circ} \mathrm{C}$. It was observed that RRF values increased with increasing purge-off time from 1.5 to $2.5 \mathrm{~min}$, then decreased with the purge-off time increasing to $3.5 \mathrm{~min}$ (shown in Figure 4). A possible reason of the decreasing RRF during the longer time is that the produced solvent "cloud" in the injector after a longer purge-off time prevented the derivatives which would be brought out by the carrier gas (Elie, Baron, \& Birkett, 2012). Thus, 2.5 min was selected 
as the optimised purge-off time that analytes got highest RRF at this value. This result agreed with Ho's study which reported 2.5 min gave the highest yield for on-line silylation process (Ho \& Ding, 2012).

\subsection{Volumes of derivatization reagent}

The volume of derivatization reagent had also a significant effect on the derivatization efficiency. The excessive of the volume of silylating reagent could disturb the analytes separation, thus led the reduction of derivatization efficiency (Toledano, Cortes, Andini, Vazquez, \& Villen, 2012). As well as the insufficient of silylation obtain poor separation due to incompletely derivatization (Basheer, Parthiban, Jayaraman, Lee, \& Valiyaveettil, 2005). In this present work, the volume of silylation reagent was varied, $0.5 ; 1.0 ; 2.0$ to $3.0 \mu \mathrm{L}$. When $0.5 ; 2.0$ and $3.0 \mu \mathrm{L}$ were used, the poor peak resolution was observed, as shown in Figure 5. Therefore, $1.0 \mu \mathrm{L}$ was selected as the best volume of silylating reagent.

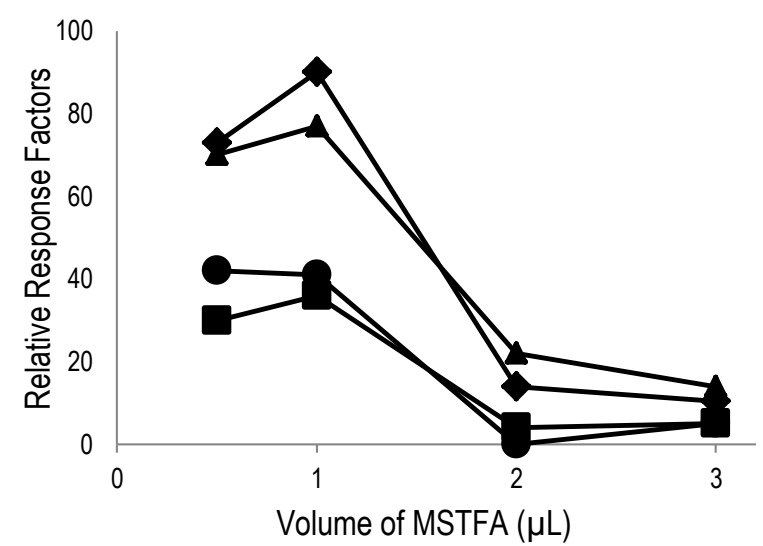

Fig. 5. Effect of the volumes of silylating. MP (• ), EP $(\boldsymbol{-}), \operatorname{PP}(\boldsymbol{\Delta})$, and $\mathrm{BP}(\bullet)$.

\section{Conclusion}

The study was focused on the optimization of on-line silylation for parabens analysis using gas chromatography-mass spectrometry. On-line silylation appears to be a promising method for parabens derivatization due to it is low cost, environmental friendly and convenient method. MSTFA (N-methyl-N-trimethylsilyltrifluoroacetamide) was selected as the most effective silylating reagent for derivatization of parabens. Many factors influenced the derivatization process, such as, injection-port temperature, purge-off time and the volume of silylating reagent were optimised, resulting the good derivatization efficiency which conditioned by $260^{\circ} \mathrm{C}$ of injection-port temperature for $2.5 \mathrm{~min}$ purge-off time (in splitless mode) and $1 \mu \mathrm{L}$ of MSTFA.

\section{Acknowledgement}

This research was supported by a grant from the Ministry of Science and Technology of Taiwan under contract No. MOST 103-2113-M-008-005MY2. The authors thank to the Instrumental Center of National Central University for the instrumentation support. We also thank to ChihChung Hsieh and Jhih-Ming Chen who helped the author to do this research.

\section{References}

Azzouza, A., Rascon, A.J., \& Ballesteros, E. (2016). Simultaneous determination of parabens, alkylphenols, phenylphenols, bisphenol $A$ and triclosan in human urine, blood and breast milk by continuous solid-phase extraction and gas chromatography-mass spectrometry. Journal of Pharmaceutical and Biomedical Analysis, 119, 16-26. doi:10.1016/j.jpba.2015.11.024

Basheer, C., Parthiban, A., Jayaraman, A., Lee, H.K., \& Valiyaveettil, S. (2005). Determination of alkylphenols and bisphenol-A: a comparative investigation of functional polymer-coated membrane microextraction and solid-phase microextraction techniques. Journal of Chromatography A, 1087(1-2), 274-282. doi:10.1016/j.chroma.2005.03.014

Bowden, J.A., Colosi, D.M., Mora-Montero, D.C., Garret, T.J., \& Yost, R.A. (2009). Enhancement of chemical derivatiozation of steroids by gas chromatography/mass spectrometry (GC/MS). Journal of Chromatogrphy B, 877(27), 3237-3242.

Elie, M.P., Baron, M.G., \& Birkett, J.W. (2012). Injection port silylation of $\mathrm{y}$-hydroxybutyrate and trans-hydroxycrotonic acid: conditions optimisation and characterisation of the ditert-butyldimethylsilyl derivatives by GCMS. Analyst, 137(1), 255-262. doi:10.1039/c1an15825b 
Ferreira, A.M., Moder, M., \& Laespada, M.E. (2011). Stir bar sorptive extraction of parabens, triclosan and methyl triclosan from soil, sediment and sludge with in situ derivatization and determination by gas chromatography-mass spectrometry. Journal of Chromatography A, 1218(25), 3837-3844. doi:10.1016/j.chroma.2011.04.055

Han, C., Xia, B., Chen, X., Shen, J., Miao, Q., \& Shen, Y. (2016). Determination of four paraben-type preservatives and three benzophenone-type ultraviolet light filters in seafoods by LC-QqLIT-MS/MS. Food Chemistry, 194(1), 1199-1207. doi:10.1016/j.foodchem.2015.08.093

Hines, E.P., Mendola, P., van Ehrenstein, O.S., Ye, X., Calafat, A.M., \& Fenton, S.E. (2015). Concentrations of environmental phenols and parabens in milk, urine and serum of lactating North Carolina women. Reproductive Toxicology, 54, 120-128. doi:10.1016/j.reprotox.2014.11.006

Ho, Y.C., \& Ding, W.H. (2012). Solid-phase extraction coupled simple on-line derivatization gas chromatography tandem mass spectrometry for determination of benzophenone-type UV filters in aqueous samples. Journal of the Chinese Chemical Society, 59(1), 107-113. doi:10.1002/jccs.201100317

Liao, C., Chen, L., \& Kannan, K. (2013). Occurrence of parabens in foodstuffs from China and its implications for human dietary exposure. Environment Intertional, 57-58, 68-74. doi:10.1016/j.envint.2013.04.001

Moos, R.K., Koch, H.M., Angerer, J., Apel, P., Schroter-Kermani, C., Bruning, T., \& Kolossa-Gehring, M. (2015). Parabens in $24 \mathrm{~h}$ urine samples of the German Environmental Specimen Bank from 19952012. International Journal of Hygiene and Environmental Health, 218(7), 666-674. doi:10.1016/j.jiheh.2015.07.005
Rocio-Bautista, P., Martinez-Benito, C., Pino, V., Pasan, J., Ayala, J.H., Ruiz-Perez, C., \& Afonso, A.M. (2015). The metal-organic framework HKUST-1 as efficient sorbent in a vortex-assisted dispersive micro solidphase extraction of parabens from environmental waters, cosmetic creams, and human urine. Talanta, 139(1), 13-20. doi:10.1016/j.talanta.2015.02.032.

Rodas, M., Portugal, L.A., Avivar, J., Estela, J.M., \& Cerda, V. (2015). Parabens determination in cosmetic and personal care products exploiting a multi-syringe chromatographic (MSC) system and chemiluminescent detection. Talanta, 143(1), 254-262. doi:10.1016/j.talanta.2015.04.055

Scott, R. P. (2003). Gas chromatography, Book 2, Chrom-Ed Book Series. Retrieved September 20, 2016, from http://www.library4science.com/eula.html2

Shanmugam, G., Ramaswamy, B.R., Radhakrishnan, V., \& Tao, H. (2010). GCMS method for the determination of paraben preservatives in the human breast cancerous tissue. Microchemical Journal, 96(2), doi:10.1016/j.microc.2010.07.005

Toledano, R.M., Cortes, J.M., Andini, J.C., Vazquez, A., \& Villen, J. (2012). On-line derivatization with on-line coupled normal phase liquid chromatography-gas chromatography using the through oven transfer adsorption desorption interface: application to the analysis of total sterols in edible oils. Journal of Chromatography $A$, 1256, 191-196. doi: 10.1016/j.chroma.2012.07.057

Tran, T.M., Minh, T.B., Kumosani, T.A., \& Kannan, K. (2016). Occurrence of phthalate diesters (phthalates), p-hydroxybenzoic acid esterx (parabens), biphenol A diglycidyl ether (BADGE) and their derivates in indoor dust from Vietnam: Implications for exposure. Chemosphere, 144, 1553-1559. doi:10.1016/j.chemosphere.2015.10.028 
Wang, Q., Ma, L., Yin, C.R., \& Xu, L. (2013). Developments in injection port derivatization. Journal of Chromatography A, 1296, 25-35. doi: 10.1016/j.chroma.2013.04.036

Wu, J., \& Lee, H.K. (2006). Injection port derivatization following ion-pair hollow fiber-protected liquid-phase microextraction for determining acidic herbicides by gas chromatography/mass spectrometry. Analytical Chemistry, 78(20), 7292-7301. doi:10.1021/ac060966e
Wu, J., Hu, R., Yue, J., Yang, Z., \& Zhang, L. (2009). Determination of fecal sterols by gas chromatography-mass spectrometry with solid-phase extraction and injectionport derivatization. Journal of Chromatography A, 1216(7), 1053-1058. doi:10.1016/j.chroma.2008.12.054 